\title{
Propuesta de modelo de Gestión para redes eléctricas con Generación Distribuida a través de Unidades de Medición Fasorial
}

\author{
Luis A. Arias(1), Edwin Rivas(2) y Luis M. León ${ }^{(2)}$ \\ (1) Facultad de Ingeniería, Programa de Ingeniería Electromecánica, Fundación Universidad Autónoma de \\ Colombia, Calle 12 No 4-32, Bogotá-Colombia (e-mail: lincarias@yahoo.com ) \\ (2) Facultad de Ingeniería, Universidad Distrital Francisco José de Caldas, Carrera 7 No 40-52 Bogotá - \\ Colombia (e-mail: erivas@udistrital.edu.co; maleonv@correo.udistrital.edu.co)
}

Recibido Jul. 27, 2016; Aceptado Sep. 29, 2016; Versión final Dic. 20, 2016, Publicado Abr. 2017

\begin{abstract}
Resumen
En el presente artículo se propone un modelo de gestión para redes de distribución eléctrica que incluyan elementos de Generación Distribuida (GD), mediante el monitoreo constante a través de Unidades de Medida Fasorial (PMU). Se analizan los conceptos de PMU, GD, algunos modelos de gestión para redes y el lenguaje de modelado Unificado UML, que es usado como base para el proceso de modelado del sistema de gestión propuesto. Posteriormente, se ilustran las etapas de modelado, los elementos estructurales y la funcionalidad que tiene el modelo de sistema de gestión propuesto. Se concluye que por tratarse de modelos de diseños abiertos y genéricos, se permite fácilmente incluir nuevos parámetros o un nuevo direccionamiento de los elementos de red, o nuevas estrategias de control para cumplir la tarea de gestión que se requiera.
\end{abstract}

Palabras clave: modelo de gestión; red de distribución eléctrica; generación distribuida; unidad fasorial de medición; UML

\section{Proposal of a management model for electrical networks with distributed generation using Phasor Measurement Units}

\begin{abstract}
In this article, a management model for electricity distribution networks is proposed. The electricity distribution network includes elements of distributed generation (DG). The network monitoring is done through Phasor measurement units (PMU). The concepts of PMU, DG, management models for networks and Unified modeling language UML are analyzed. The UML language is used as the basis for modeling process management system proposed. Later, stages of modeling, structural elements and functionalities of the proposed model management system are illustrated. It is concluded that being model of open and generic design it can be easily include new parameters, or change de direction of the network elements, or new control strategies that may be needed to fulfill a desired management task.
\end{abstract}

Keywords: management model; electrical distribution network; distributed generation; phasor measurement unit; UML 


\section{INTRODUCCIÓN}

La gestión en una red de distribución eléctrica puede interpretarse como el conjunto de operaciones que se realizan para administrar las tareas de la red, y que implica la coordinación entre los eslabones o elementos estructurales de la red, orientándose a dar respuesta a una serie de funciones específicas, que para el presente trabajo se denominan funciones objetivo (Inglis, et al., 2010; Zhang et al., 2011). El manejo de la red de distribución con la inclusión de elementos de generación distribuida supone algunos inconvenientes para una correcta gestión y control desde el punto de vista técnico. Los inconvenientes a los que se hace alusión radican en el hecho de que la producción de energía eléctrica no es constante debido a que se fundamentan en fuentes renovables caracterizadas por la aleatoriedad de los recursos en cuanto a tiempo y disponibilidad como la solar, eólica, biomasa entre otros. Otros inconvenientes ligados a la inclusión de elementos de GD, son por ejemplo, el desbalance de potencia y tensiones durante el abastecimiento de cargas; la presencia de "imperfecciones" en la calidad de la señal suministrada tales como flickers, slags, e incrementos en el THD (Total Harmonic Distorsion) en voltaje, por encima de los niveles admisibles de acuerdo a la normativa IEEE-519 (Masoum et al., 2007; Ye, et al., 2015).

Las definiciones para GD son variadas en razón a la multiplicidad de los factores que son tomados en cuenta al momento de definirla, como por ejemplo, las tecnologías de generación de energía utilizadas, los límites de potencia, las conexiones a la red eléctrica y las distancias al usuario final (Dulău et al., 2014; Dulău et al., 2015; Jagtap y Khatod, 2016). En un primer acercamiento la GD se conoce como cualquier tecnología de generación que provee electricidad a pequeña escala a usuarios que se encuentran distantes de las redes de generación centralizada; y que puede conectarse de forma directa al consumidor, a la red de distribución o incluso a la de transmisión (Bayod-Rújula, 2009). Otra definición es dada por la Agencia Internacional de Energía, que afirma sobre la GD como el conjunto de elementos de generación alternativa que se conecta a la red de distribución de bajo voltaje y asociado a tecnologías tales como generadores eléctricos, mini y microturbinas a gas, celdas de combustible, colectores solares, sistemas fotovoltaicos y generadores diésel (International Energy Agency, 2013). De acuerdo a la EPRI (Electric Power Research Institute) la GD es interpretada como "El uso de tecnologías de generación modular de pequeña capacidad, dispersa a la largo del sistema de distribución" (McGranaghan, 2009).

Dentro de las fuentes de energía que pueden usarse en GD se tienen generadores tradicionales y no tradicionales. Los generadores tradicionales usan motores de combustión, turbinas de baja velocidad, motores diésel, turbinas reciprocantes y microturbinas. Los generadores no tradicionales usan dispositivos electroquímicos como las celdas de combustible, dispositivos de almacenamiento como las baterías y los flyweel o almacenadores de energía rotacional y otros dispositivos de energía renovables como las turbinas eólicas, las celdas solares, la biomasa y las pequeñas centrales hidroeléctricas o PCH (Mohamed, 2008).

Los flujos de Potencia en la red son influenciados por las características de las fuentes de suministro, los consumidores y la impedancia de los circuitos de conexión. Cuando las unidades de GD son introducidas a la red de distribución se presenta un cambio esencial y es que los flujos unidireccionales para los cuales fueron diseñadas las redes son ahora de tipo bidireccional. Estos flujos bidireccionales añaden cierto nivel de incertidumbre en la dirección y magnitud de los flujos totales en la red de distribución y pueden suponer un significativo riesgo en la seguridad y comportamiento de la red en general (Dolan et al., 2013). El desarrollo actual de las redes eléctricas enfocado en las GD tales como la solar, eólica y biomasa entre otras implica un análisis integral de aspectos como el económico, tecnológico, social, medioambiental y legal que debe ser desarrollado en forma jerárquica. Este tipo de enfoque jerarquizado debe ser base para el desarrollo de las redes futuras (Sadeghi et al., 2012). La inclusión de GD trae beneficios a las redes eléctricas tales como la reducción de los flujos de potencia a través de las líneas en redes de transmisión y distribución, aminora los problemas de crecimiento de la carga, reduce las pérdidas en transmisión y distribución, además de mejorar la calidad del servicio y la confiabilidad del sistema de distribución.

Sin embargo, de acuerdo a la visión de otros autores se señala que la instalación de varias GD puede afectar la calidad de la energía y confiabilidad de los Sistemas eléctricas convencionales de forma negativa. Por ejemplo, las fuentes de generación como la solar y eólica incluyen elementos inversores de corriente directa a corriente alterna, los cuales son generadores de señales armónicas, que complican la normal operación de los dispositivos de regulación de voltaje; a su vez pueden ocasionar la existencia de parpadeos en la red, y pueden cambiar la operación normal de la protección que opera para eliminar fallas de sobre corriente y cortos circuitos (Tse y Zhou, 2009; Muñoz-Galeano et al., 2016; Alcalá et al., 2014).

En la protección del sistema de distribución, se debe considerar que la corriente de carga suministrada por el GD puede modificar el alcance del equipo de protección mediante la variación de la sensibilidad de los instrumentos, causando una corriente más alta que la necesaria para la reacción del sistema de protección. Además de la variación en alcances de la protección, la GD aporta no linealidad al comporta-miento del 
sistema y reduce la confiabilidad de las protecciones por lo cual se requiere una continua adaptación a escenarios dinámicos de gran variabilidad. En la actualidad se cuenta con algunos desarrollos de algoritmos clásicos de optimización para la gestión de la red con GD que incluyen eslabones de control encargados de la desconexión y reconexión de cargas y de ser posible su deslastre (Ms, et al., 2012; Ruiz-Romero et al., 2014). Asimismo se han desarrollado algoritmos para la planificación de las redes de distribución, incluyendo GD, que proponen la gestión de la red de distribución a través de funciones multi-objetivo cuya solución se realiza mediante algoritmos genéticos (Tazvinga et al., 2014; Nascimento et al., 2015).

\section{GESTIÓN EN REDES ELÉCTRICAS CON GD}

En el área de desarrollo de sistemas de gestión es posible encontrar varios modelos diseñados para el control y manejo en general de las redes de distribución con inclusión de GD. La gestión de los sistemas de distribución debe considerar las unidades de GD en su funcionamiento, niveles de potencia, la ubicación dentro de la red y niveles de priorización entre otros parámetros. Uno de los aspectos más importantes en la gestión está vinculado a la obtención de la máxima capacidad de potencia de la unidad de GD y que no se vea afectada negativamente por el funcionamiento de otras unidades de GD en la red. La tarea de gestión para varias unidades de GD no es sencilla y se ha abordado por algunos autores en técnicas de optimización de flujo en sistemas de energía de configuración adaptable (Kaabi, et al., 2014).

Uno de los aspectos más importantes es el análisis de los impactos de la GD sobre el perfil y las pérdidas de los sistemas de redes de distribución de tensión, debido a la diversidad de características de inyección de potencia en la red que tienen los elementos de GD. Por ejemplo, existen unidades de GD que inyectan potencia activa, otros absorben potencias reactivas y algunos tienen un comportamiento hibrido. De este comportamiento diverso de las GD, surgen investigaciones que evalúan la inclusión gradual de la GD con diferentes porcentajes de penetración en la red de distribución (Jagtap y Khatod, 2016). Para procesos de transferencia de potencia activa y reactiva entre sistemas de corriente alternada interconectados, que bien pueden ser el de la red y aquello ligados a sistemas de GD, se han propuesto convertidores BTB (Back To Back) que actúan desde baja potencia hasta alta potencia. Los BTB permiten controlar de manera independiente el flujo bidireccional de potencia activa y reactiva, facilitando así la gestión de los flujos de potencia entre sistemas de distribución eléctrica convencionales y aquellos apoyados en GD (Alcalá et al., 2014).

La alta penetración de elementos de GD como los vehículos eléctricos que se prevé para los próximos años, se estudia en trabajos como los de (You y Segerberg, 2013), donde se analiza la integración de recursos energéticos distribuidos en las redes de distribución de baja tensión. Los recursos distribuidos se utilizan para la carga de vehículos eléctricos a pequeña escala, pero deben tenerse en cuenta las restricciones termodinámicas de las cargas y como pueden influenciar la red, lo cual debe considerarse en los nuevos sistemas de gestión de red de distribución (You y Segerberg, 2013). En trabajos como los de Abdullah, son desarrollados modelos de despacho, basados en el análisis de la topología de la red, y la medición del flujo antes y después de conectar o desconectar una carga, los mecanismos de desconexión y transferencia de carga. Las caídas de tensión se evalúan a través de los índices cuantitativos calculados con diferentes grados de contingencia (Abdullah, 2011), permitiendo así evaluar la seguridad y la vulnerabilidad del sistema, ya sea con métodos convencionales de evaluación o con métodos alternativos de estimación como aquellos basados en redes neuronales (Patricia et al., 2013).

De otra parte, se han desarrollado algoritmos adaptativos para la planeación de redes de distribución con GD, donde se proponen funciones multi-objetivo, resolviendo algoritmos genéticos (Shi et al., 2011). En la gestión en las redes de distribución que se enfoque a la integración de consumidores activos, y elementos de generación distribuida, resulta relevante la propuesta alrededor del desarrollo de algoritmos adaptativos que respondan a funciones objetivo variables en el tiempo y con criterios de evaluación que van desde lo técnico, económico e incluso social, legal y ambiental (Sadeghi et al., 2012; Di Somma et al., 2015; Sismotto y Hage, 2005). En la revisión de la literatura, se encuentran propuestas de gestión en tiempo real para sistemas eléctricos de potencia, basados en nodos piloto. Los nodos piloto hacen parte de sistemas de control experto, que permiten, ante contingencias, realizar un recalculo de la matriz de sensibilidad de la red eléctrica. La aparición de contingencias como desbalances de flujo de carga es habitual al interconectarse sistema de GD en la red. Un aspecto de gran relevancia en la gestión es la detección de fallas al incluirse los elementos de GD (Gómez et al., 2012).

Un sistema de Gestión Energética es aquel que permite que una red mantenga un ciclo continuo de planificación, implantación, verificación y mejora de las acciones que se llevan a cabo para el cumplimiento de sus obligaciones energéticas. La gestión permite fomentar la Eficiencia Energética de las empresas con su correspondiente ahorro económico y energético, logrando de esta manera un impacto positivo en indicadores medioambientales ligados a la disminución de gases de efecto invernadero (Di Somma et al.,2015; Kaabi et al., 2014; Naus, et al., 2014). 


\section{MODELOS DE GESTIÓN EN REDES CON GD}

En los siguientes párrafos se abordaran algunas de las estrategias de gestión para las fuentes de GD a nivel tecnológico como son los sistemas SCADA (Supervisory Control And Data Acquisition), las microrredes y las Plantas Virtuales de potencia (VPP).

Los sistemas SCADA son utilizados en labores de gestión, recibiendo datos de dispositivos sensores que monitorean el estado de las subestaciones y dispositivos de protección que puedan alterar sus alcances en función a la conexión o desconexión de elementos GD, ya sea aguas arriba o aguas debajo de los barrajes principales, y que resulta imprescindible para índices altos de penetración de GD en la red (Nair y Zhang, 2009) . Los SCADA son utilizados en la planificación de las redes asignando la potencia eléctrica óptima a cada unidad de generación, minimizando los costes de funcionamiento de producción. Cuando la producción de energía se realiza gracias a un sistema híbrido con fuentes renovables y convencionales, la reducción al mínimo de los costes funcionales conduce a la minimización de la energía convencional (Figueiredo y Martins, 2010).

En la actualidad una buena parte de la gestión de redes que incluyen GD a gran escala y evalúan los impactos de los mismos, se apoya en sistemas SCADA compuestos básicamente por software para la toma de decisiones, interfaces hombre-máquina con módulos de accesibilidad y controles locales y remotos para las comunicaciones, a menudo soportados en redes Ethernet. Los principales objetivos de los SCADA con la vigilancia y el control de la tecnología a partir del seguimiento de los parámetros eléctricos y no eléctricos del sistema híbrido de la producción y el consumo de electricidad a partir de fuentes de energía renovables, el control sobre los elementos de toma de decisiones implementado dentro del sistema de supervisión y el control del sistema (Dumitru y Gligor, 2014).

En el proceso de integración de GD un aspecto importante es el monitoreo y control de los dispositivos de almacenamiento, sus acoples inversores y los parámetros transitorios al momentos de conexión y desconexión a la red de dichos inversores. Para la coordinación de estas labores los sistemas SCADA resultan ser una herramienta que ofrece gran versatilidad (Dulău et al., 2015). Algunas soluciones analizan a los generadores distribuidos y sus cargas asociadas como un subsistema denominado microrred (Lasseter, 2007). Una microrred es un sistema discreto de energía eléctrica a pequeña escala, que puede estar desconectado (modo aislado) o conectado a la red (modo interconectado) y consiste en fuentes renovables y tradicionales interconectadas entre si y contando con sistemas de almacenamiento ya sea a través de baterías o la gestión de la energía en los edificios inteligentes (Soshinskaya, et al., 2014).

Según De Brabandere et al (2007) la gestión de la red de distribución que integra GD, se puede realizar por medio de microrredes desde la perspectiva de redes autónomas de energía caracterizadas por alto nivel de penetración de recursos distribuidos de energía, que participan en un mercado de precios en tiempo real. La operación de estas redes autónomas se realiza sin un operador central, y se fundamenta en las redes públicas de comunicación. Las micro-redes resultan una estrategia bastante interesante sobre todo si se muestran como soluciones integrales para energía no solo de suministro eléctrico, sino también de gas y calefacción (Ramírez et al, 2013).

Autores como Karabiber et al (2013) y Palizban et al (2014) analizan la gestión de la microrred enfocándose en el problema de mantener la sincronización de la microrred una vez esta pasa de modo interconectado a modo aislado, que resulta vital en el momento de evaluar la estabilidad de los puntos de operación una vez sea necesaria una reconexión posterior. Para este caso la gestión es soportada en la observancia de las restricciones técnicas para la interconexión de elementos de GD a la red de distribución y que son tratados ampliamente en el protocolo IEEE 1547 (Basso y DeBlasio, 2011). Al integrar GD mediante microrredes debe analizarse el impacto de la distribución de costos y beneficios entre los agentes participes del mercado: el administrador de la microrred, la red eléctrica regulada, los clientes dentro de la microrred y los clientes que están fuera de la microrred. Una herramienta de análisis usada en microrredes es la teoría de juegos cooperativos donde se evalúan diferentes escenarios de sinergia entre los agentes del mercado (Lo Prete y Hobbs, 2016).

La VPP se define como un nuevo modelo de infraestructura de la energía, consistente en la integración de diferentes unidades de generación distribuida en una red de generación de energía controlada por un sistema central de gestión de la energía (Bayod-Rújula, 2009). La VPP combina fuentes tanto renovables como no renovables de energía, al igual que dispositivos de almacenamiento y usuarios no necesariamente conectados entre sí, que son reunidos para salir al mercado y aparecer como un todo, como una planta de energía con una salida horaria definida y unas posibilidades propias de generación que le permiten incluso ofertar en un mercado mayorista, siendo incluidos en procesos de despacho horario diario o de mediodía (Pudjianto et al., 2007; Bayod-Rújula, 2009; Giuntoli y Poli, 2013). 
Andersen et al (2008) presentan un modelo de gestión para la integración de GD, apoyado en VPP analizado a partir de arquitectura de software orientada a servicios. Se proponen tres escenarios: uno inicial donde la VPP se muestra como eje central donde convergen los elementos de recursos energéticos distribuidos (DER), que comprenden los elementos de GD, dispositivos de almacenamiento y controladores para cargas puntuales. Luego es propuesto un escenario intermedio, donde se tienen varias VPP auxiliares, que gestionan los DER de cada microrred, y que a su vez son controlados por una VPP central; y un tercer escenario, donde se presenta un sistema de servicios centralizado que involucra los servicios de la VPP y gestiona la información del entorno externo a la red como estado de las troncales adyacentes a cada microrred, el estado meteorológico y sus previsiones para las fuentes de GD y los precios de la energía en tiempo real.

Las VPP pueden utilizarse como estrategias de integración para unidades descentralizadas de tipo eólico y fotovoltaico, ofreciendo multiplicidad de servicios para un pool de mercados de electricidad, abordada mediante un análisis técnico- económico de cada unidad de GD y su consumidor asociado. La versatilidad de las VPP permite que integren además de usuarios poseedores de DER elementos de la red como Plantas de procesamiento de residuos permitiendo establecer clúster económicos y energéticos en el marco de proyectos económica, social y medioambientalmente sostenibles (Arias, Rivas y Vega, 2014; Schäfer et al, 2015; Sowa et al, 2014) Las VPP facilitan la integración de los DER, permitiendo subsanar en gran medida inconvenientes ligados a la incertidumbre en el comportamiento de los elementos DER como la GD, gracias al manejo paralelo de tecnologías asociadas a sistemas de almacenamiento, y mecanismos de RD. La facilidad de adaptación de las VPP permite que participen en mercados de tipo DAM (Day Ahead Market: Mercado de día anterior) y en mercados de tipo BM (Balancing real time market: mercado de balance en tiempo real; Dietrich et al, 2015).

\section{PMU (PHASOR MEASUREMENT UNIT)}

Una PMU provee las magnitudes de los fasores de voltaje y corriente con sus respectivos ángulos de desfase, calculados a partir de una referencia sincronizada que es provista por un receptor GPS (Sistema de Posicionamiento Global). Este receptor GPS mejora las velocidades de muestreo de seguimiento en tiempo real de cada uno de los elementos del sistema eléctrico, facilitando la toma de decisiones, posibilitando adelantar estudios de comportamientos futuros de la red, y en general registrar todos aquello cambios que no se pueden grabar con la monitorización convencional (registradores de falla y analizadores de calidad)(Ruschmann, Wu y Huang, 2012), (Ren, Kezunovic, y Guan, 2012). Las PMU realiza la estimación de los fasores de voltaje y corriente, al igual que su frecuencia y su deriva, permitiendo la detección de disturbios eléctricos en la red de distribución tales como fallas, cambios intempestivos en la carga, fluctuaciones como sobre cargas, flujo inversos o la presencia de armónicos (Abur y Galvan, 2012).

El desarrollo de nuevos modelos de gestión para redes de distribución implica trabajar en el rediseño de dispositivos existentes para medición tales como los PMU, los monitores de gestión como los fasores de corriente, voltaje, cambios de frecuencia, los flujos de potencia activa y reactiva entre otros. Las redes de distribución con elementos de GD son caracterizadas por un alto dinamismo, implicando grandes requerimientos para los equipos de medida en cuanto a capacidad de procesamiento y respuesta en tiempo real, en procura de incrementar la capacidad de almacenamiento y la inclusión de sistemas de gestión para responder a las funciones objetivo previamente establecidas (Ruiz-Romero et al., 2014). La utilización de PMU ayuda en la localización de fallas en sistemas de distribución. La localización de fallas en los sistemas de distribución es una labor compleja debida a la presencia de conductores no homogéneos, cargas intermedias laterales, y el desbalance de las cargas ((Gómez et al., 2012)(Gil et al, 2014).

Para la propuesta de gestión se ha sugerido a nivel de hardware emplear un prototipo portable de PMU a bajo costo y con alta capacidad de información, que permita hacer un seguimiento a los cambios continuos en los flujos de red. En cuanto a nivel de software, se propone trabajar con aplicaciones tipo WAMS (Wide Area Measurement System- Sistemas de medida de área ancha) para la gestión de la red; y finalmente, a nivel de comunicaciones emplear sistemas de protocolo abierto, tanto para medios guiados como no guiados tipo GPRS (General Packet Radio Service), ETHERNET, WIFI, o BPL (Broadband Power Line) sin considerar canales de comunicación dedicados (Pal y Thorp, 2012; Mukhopadhyay y Chawla, 2014).

\section{UML (UNIFIED MODELING LANGUAGE)}

UML es un lenguaje de modelado universal que permite utilizar la descripción genérica de componentes de arquitectura y especificar por ejemplo los componentes internos de algunas estructuras y las secuencia de ensambles y construcción utilizando diagramas de clases y de secuencias (Basile, Chiacchio y Del Grosso, 2009). La formalización de los diagramas del UML permite que cada uno de estos modelos de sistemas se 
refine, permitiendo la inclusión y la refinación de las relaciones entre los elementos, chequeando la consistencia interna de cada uno de los elementos, y verificando la interconexión entre los elementos.

UML surge como una herramienta de gran aceptación cuando es necesario soportar el diseño y la implementación de una solución automatizada, que subyace en un modelo de gestión de cualquier sistema, para ello se debe tener la documentación apropiada para su desarrollo y su mantenimiento subsiguiente o eventuales modificaciones. Lo anterior resulta deseable y debe tenerse en cuenta en las representaciones visuales del sistema para su adecuada operación y un mejor entendimiento de los diseños (Basile et al., 2009). La principal ventaja de UML es que constituye un lenguaje de propósito general, lo cual en ocasiones puede verse como una leve desventaja, en razón a que no puede representar en toda su dimensión el detalle de cada situación y las características propias de los dominios específicos (Vega et al, 2014). No obstante, la universalidad del UML permite a los desarrolladores enfocarse en las soluciones de automatización desde perspectivas y aspectos más relevantes para su proceso creativo, tales como el establecimiento de las relaciones de causa y efecto entre las variables y parámetros de una planta de procesos, identificando su comportamiento dinámico en el tiempo.

A través de UML se pueden observar las interacciones de los elementos de un sistema de gestión, como eslabones estructurales con funciones específicas, lo cual resulta de gran ayuda para el diseño de los objetos y sus subrutinas. Las subrutinas van ligadas a los parámetros y comportamientos definidos en UML y se programan luego en diversas plataformas tecnológicas como los microcontroladores, los controladores lógicos programables (PLC) y computadores personales (Arias, Garcia y Rivas., 2013). En el presente trabajo se realizaron los siguientes diagramas: Un diagrama de Caso de Uso para modelar los escenarios convencional y alternativo de la gestión; las partes estructurales del sistema de gestión y su caracterización funcional son diseñadas mediante diagramas de Clases; y finalmente, las relaciones entre los diferentes eslabones 0 estructuras del sistema son descritas a través de un diagrama de secuencias que sigue la metodología UML.

\section{MODELO DE GESTIÓN PROPUESTO}

En la figura 1, se ilustran las etapas de desarrollo del modelo de gestión que se propone. Como se observa en el diagrama de la figura 1, la primera fase del proceso de modelado inicia con el diseño de una red de área local (LAN: Local Area Network), esta red enlazara a los dispositivos de medida PMU, a los elementos de GD, a usuarios de la red y a subestaciones que estén presentes en la red, para lo cual se les asignara una dirección particular de red a través de un protocolo como ETHERNET, DHCP o similar. La segunda fase del modelado, se centra en el diseño de las funciones objetivo que tendrán como base criterios y restricciones de tipo técnico, socio-económico y medioambiental. Las funciones objetivo se estructuran de manera genérica para optimizar un determinado grupo de parámetros. En la fórmula 1, se muestra de manera genérica la forma que tienen las funciones objetivo que orientan la gestión de la red.

$\operatorname{Min} f\left(x_{1}, x_{2}, \ldots x_{n}\right)$

s.a. $\mathrm{x}_{\mathrm{i}} \leq \mathrm{N}$

Dónde: $f\left(x_{1}, x_{2}, \ldots x_{n}\right)$ es la función objetivo ligada al criterio que se quiere optimizar; $y \quad x_{i} \leq N$ es el conjunto de restricciones ligadas a la función objetivo que se quiere explorar o analizar. A manera de ejemplo, puede requerirse o bien un menor costo de energía para los usuarios, o mantener flujos de potencia reactiva dentro de límites admisibles para la red, de acuerdo al factor de potencia pactado para algunos usuarios.

La tercera fase está ligada al desarrollo de estrategias de control que responden a los resultados obtenidos en el proceso de optimización de las funciones objetivo. A manera de ejemplo, el hecho de alcanzar un mínimo precio en una función objetivo de coste de energía para la red, requiere que se desconecten una buena parte de las fuentes de GD, que tengan potencias por debajo de un límite mínimo de generación que se ha fijado para la red. En otros casos, se puede requerir que se disminuya el consumo energético de determinadas cargas de manera gradual para lo cual de manera escalonada se desconectan algunos dispositivos propios del usuario como elementos de calefacción, luminarias u otros dispositivos que se consideren factibles a una interrupción momentánea en su suministro.

En la fase final del proceso de modelado se propone diseñar algoritmos de gestión, para los cuales se propone incluir niveles de priorización o prelación de determinadas cargas, a fin de garantizar su abastecimiento o autoabastecimiento en todo momento, incluso bajo condiciones adversas como fallos o apagones en la red de distribución. De igual forma el desarrollo de algoritmos de gestión implica que se evalúen los cruces entre funciones objetivo focalizadas en los diferentes criterios antes mencionados. Por último y no menos importante, los algoritmos de gestión deben coordinar la forma como se realiza la comunicación entre los elementos constitutivos de la red de distribución: usuarios, elementos de GD, equipos de medida PMU y el administrador del sistema de gestión. 


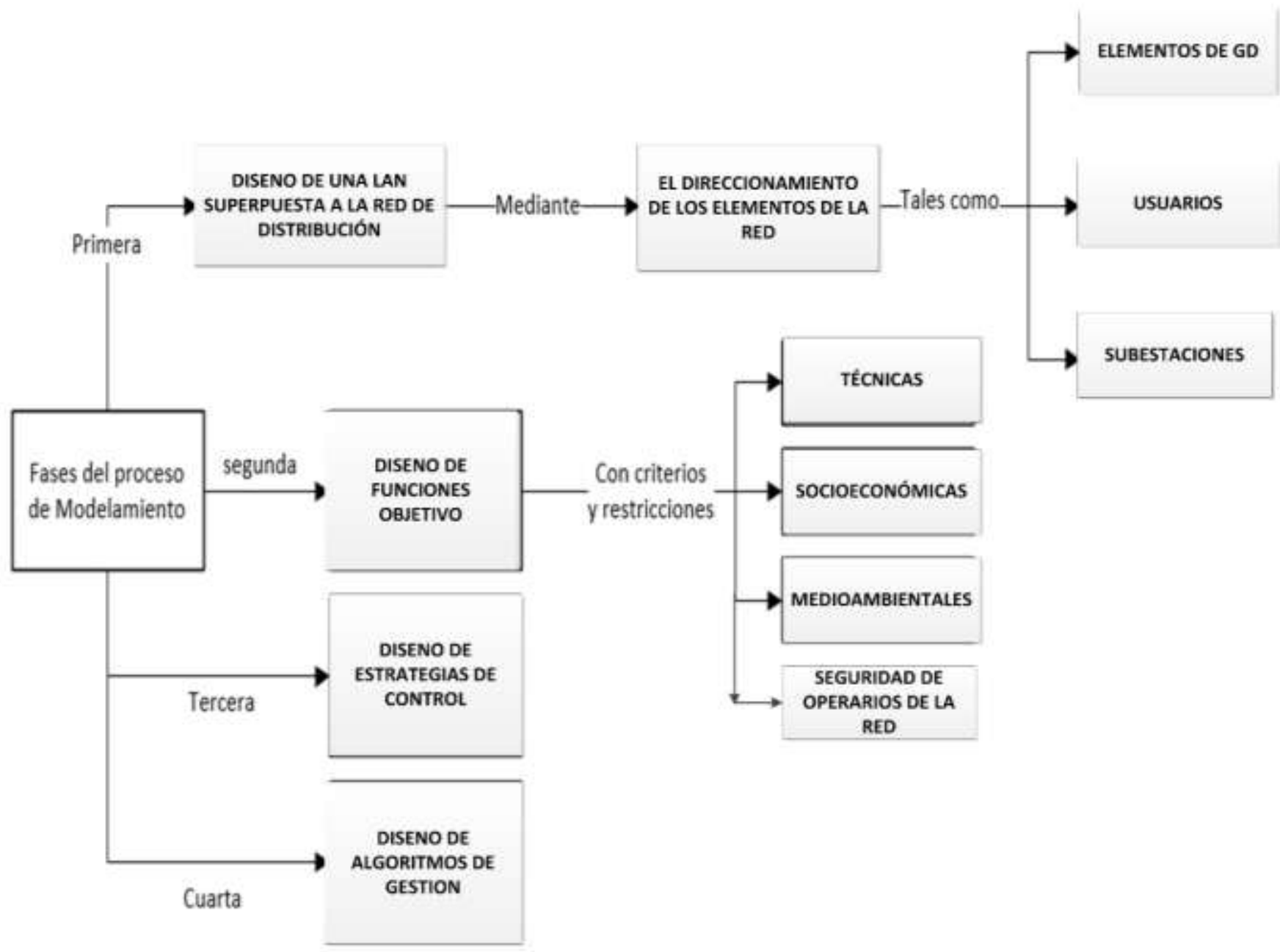

Fig. 1: Diagrama ilustrativo de las fases en el proceso de modelado del sistema gestión.

El modelo de gestión propuesto involucra aspectos técnicos, tecnológicos, económicos y medioambientales a tener en cuenta en una red de distribución, los cuales a su vez están supeditados a criterios particulares fijados por la legislación sobre la regulación y los niveles de prioridad para ciertos usuarios en determinados sectores de una región. La operación del sistema de gestión propuesto se realiza seleccionando una de las funciones objetivo: con criterio económico, técnico, socio económico o medioambiental. Debe clarificarse que los autores estiman que pueda realizarse una combinación de funciones objetivo, y la selección de niveles de prioridad para determinados grupos de usuarios. A manera de ejemplo, en el modelo de sistema de gestión se puede seleccionar que se busque un mínimo precio por kWh, y esto puede significar entonces que las fuentes de GD se avoquen a vender su energía a la red, pero esto sucederá siempre y cuando se garantice el abastecimiento pleno para usuarios de un centro de salud o de alguna entidad gubernamental que se encuentren en la red de distribución. Como se observa en el diagrama de Caso de Uso del sistema de gestión, el administrador de la red recibe información de la red de PMU, las notificaciones de cambios en la topología de la red que se deben a la conexión de elementos de GD, o por la aleatoriedad en la conexión de elementos de carga para vehículos eléctricos. De igual forma el administrador recibe información sobre los niveles de prioridad de ciertas cargas especiales tales como centros de salud, escuelas o edificios estatales.

Las PMU realizan un monitoreo continuo de los parámetros de cada segmento y elemento de la red de distribución, y luego realiza su posterior procesamiento y almacenamiento. El almacenamiento y procesamiento de los datos permite al administrador de la red realizar un control y gestión integral de la red, con generación de alarmas asociadas al mantenimiento preventivo de la red, con avisos ligados a los niveles de prioridad de los elementos de la red, sus restricciones límites de potencia de consumo o de generación, la cargabilidad de elementos como transformadores y sus líneas respectivas. De la misma forma el administrador de la red recibe información de tipo económico como los precios por kW en horas pico, los precios por kW en horas valle. Dentro de los parámetros técnicos se recibe el nivel de calidad de voltaje, los flujos de potencia activa y reactiva, el cambio en los alcances del sistema de protecciones entre otros parámetros. 


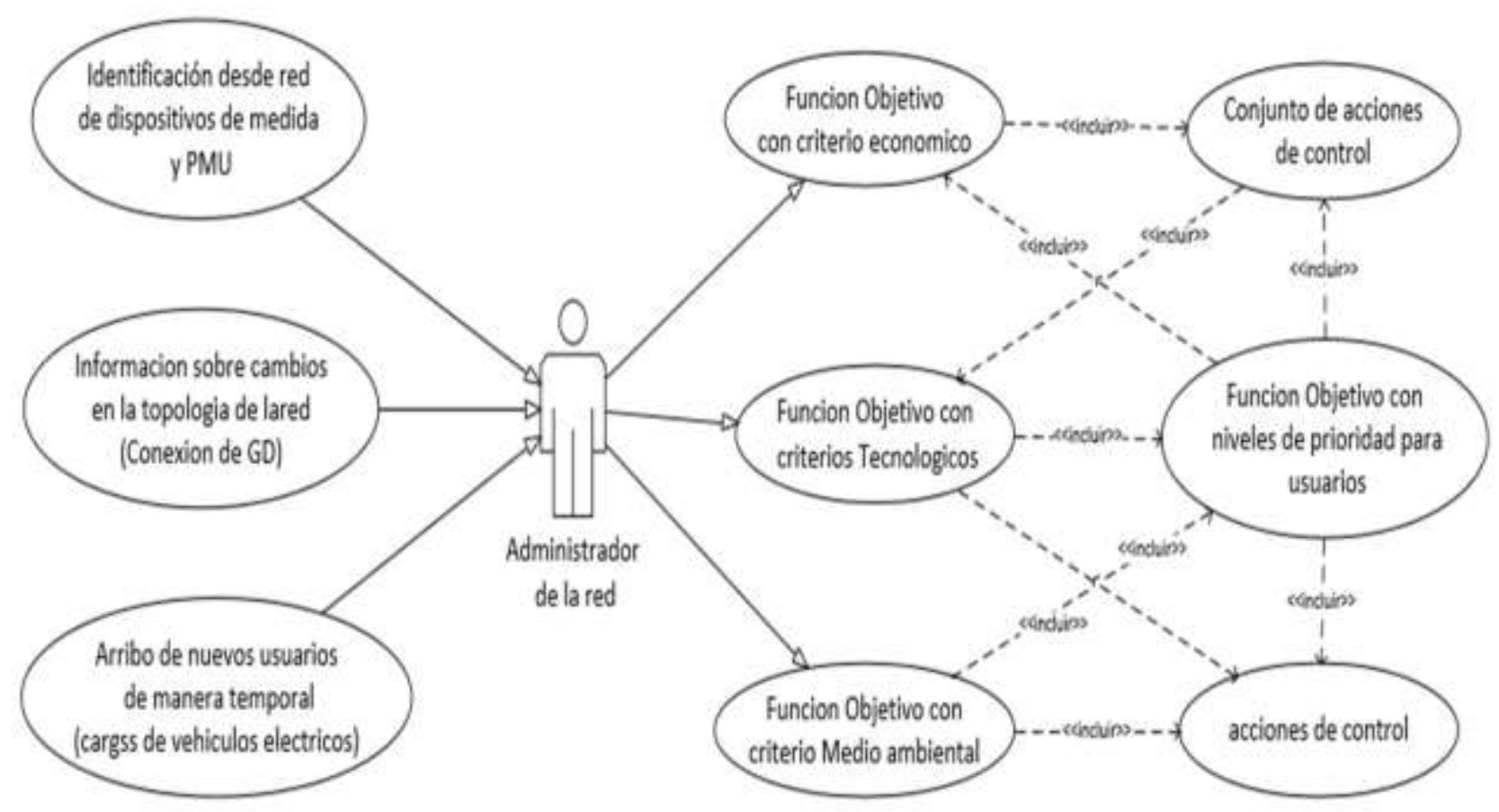

Fig. 2: Diagrama de Casos de Uso para el modelo de Gestión propuesto.

La figura 3, muestra a manera de ejemplo, el diagrama de clases para la función objetivo con criterio económico. En este caso la función objetivo busca minimizar el precio por kWh y aplanar la curva de demanda en respuesta a los altos niveles de demanda durante horas pico. Las variables a considerar son el precio en horas valle, el precio en horas pico y el porcentaje de aplanamiento de la curva de demanda que se quiere obtener al final de un determinado tiempo. Algunos de los procedimientos sugeridos para la gestión son la conexión secuencial de elementos de GD, la conmutación (conexión/desconexión) de microrredes con alto consumo y el deslastre de carga en algunas zonas con nivel de prioridad baja.

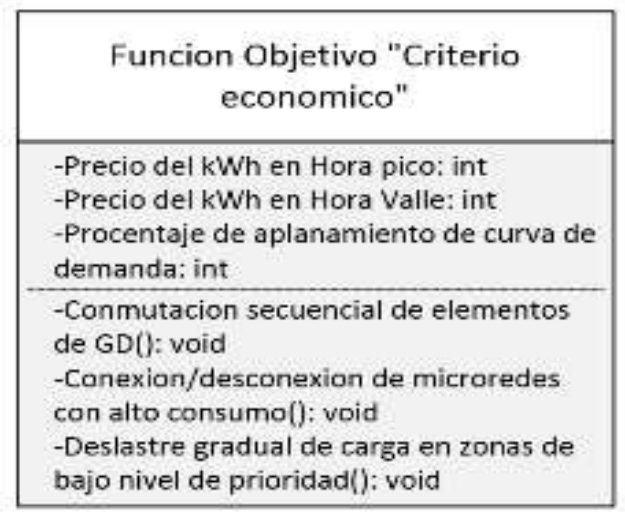

Fig. 3: Diagrama de Clases para la función objetivo con criterio económico.

El sistema de gestión propuesto opera a partir de los niveles de prioridad establecidos para cada usuario y de las funciones objetivo por cada criterio señalado anteriormente, de manera coordinada. Por ejemplo, si se abastecer un grupo de usuarios con alto nivel de prioridad, un centro hospitalario, y se selecciona trabajar con la función objetivo con criterio medioambiental, la acción a realizar implica que se conecten la mayoría de elementos de GD que se dispongan en la zona dada de la red, independientemente que la forma de la señal de voltaje no sea la mejor, y que la razón de potencia activa y reactiva este por fuera de lo deseable en ese momento. De la misma forma, si la función objetivo a usar se centra en el criterio económico y busca el menor precio del mercado para todos los usuarios de la red, la acción a tomar será desconectar una buena parte de los elementos de GD que proporcionen bajos niveles de potencia y proveerse de energía, únicamente, de los distribuidores que son alimentados de fuentes convencionales de energía. En la figura 4, se muestra la 
estructura del sistema de gestión propuesto, donde las PMU ocupan un lugar principal para el monitoreo de cada elemento de la red, en el caso concreto se observa como un elemento de GD que está incluido en un segmento de red de distribución.

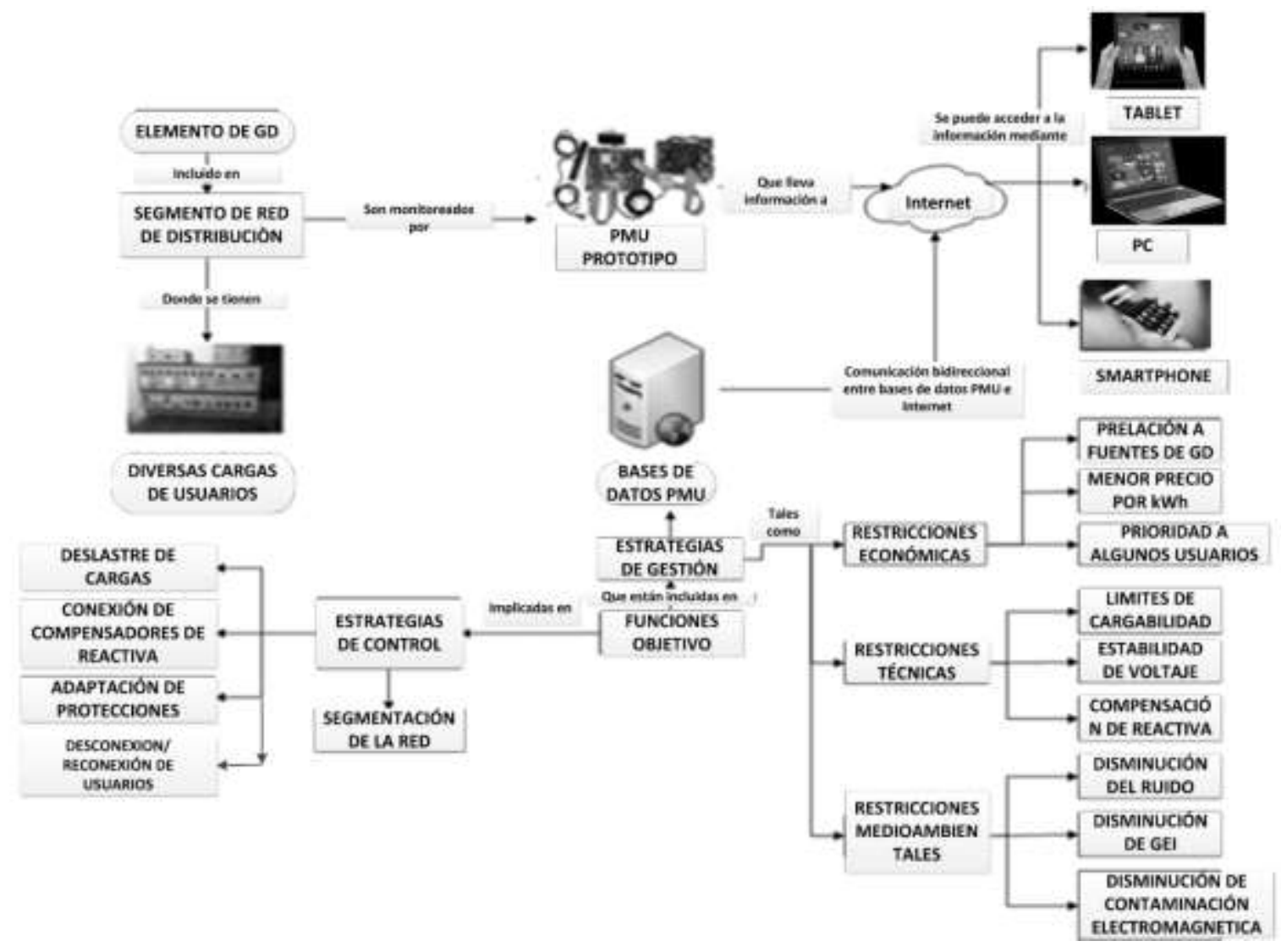

Fig. 4: Diagrama de modelo de gestión de red propuesto con PMU, para el monitoreo de un elemento de red.

Como se detalla en el diagrama de la figura 4, un elemento de la red, para el caso concreto una fuente de GD, es monitoreado a través de una PMU. La PMU lleva los parámetros característicos del elemento y de su segmento de red hacia una aplicación en Internet, un web Service, que sirve como apoyo para el sistema de gestión propuesto. A este web service se puede acceder desde diversos medios como Smart Phone, Tablet y ordenadores personales, a fin de que los administradores, y algunos usuarios con prelación puedan tener información sobre el estado de la red en tiempo real. Como se observa, en la aplicación de web Service se tiene acceso bidireccional a la base de datos de la PMU. La base de datos PMU almacena la información y realiza actualizaciones permanentes; y para casos de pérdidas de comunicación con una PMU tiene la posibilidad de realizar la gestión a partir de históricos mientras se da logra restablecimiento de la comunicación con la PMU. Las estrategias de gestión, sus funciones objetivo, las estrategias de control hacen parte de toda la estructura funcional del web service que puede dejarse abierto en su acceso a través de Internet, o bien puede alojarse en un servidor in situ. Las estrategias de control sugeridas para el sistema comprenden el deslastre de carga, la conexión de dispositivos compensadores de potencia, la adaptación de las protecciones, la desconexión/reconexión de usuarios y la segmentación de la red, en casos que se considere necesario, tales como impedir que se extienda una falla a todo el sistema, al conectarse un fuente de GD por ejemplo. El mecanismo de segmentación de la red tiene en cuenta la demanda del sistema por sectores a fin de que cuando se presente una falla se segmente o seccione esa parte del resto de la red.

En la figura 5, se ilustra el diagrama de secuencia que muestra la forma de operación del modelo de gestión propuesto para la red de distribución. Inicialmente, se toma la información de la red de PMU, luego el administrador de la red analiza los datos y genera unos indicadores de estado para los elementos de la red, a su vez genera avisos sobre la función objetivo que está operando en el momento dado y muestra el estado general de los flujos de potencia en la red de distribución. En el sistema de gestión se permite seleccionar la función objetivo con que se quiere trabajar y los parámetros de la misma que se consideren más relevantes. Una vez que la selección se realiza se envían comandos para la operación de los controladores de la red y ejecutar estas operaciones en la red respectiva que lleve a que los elementos en su accionar sean modificados; consecuentemente, las PMU registran las medidas de los cambios realizados y envían al sistema de gestión los nuevos parámetros y despliegan ante el administrador las nuevas condiciones de la red de distribución. 


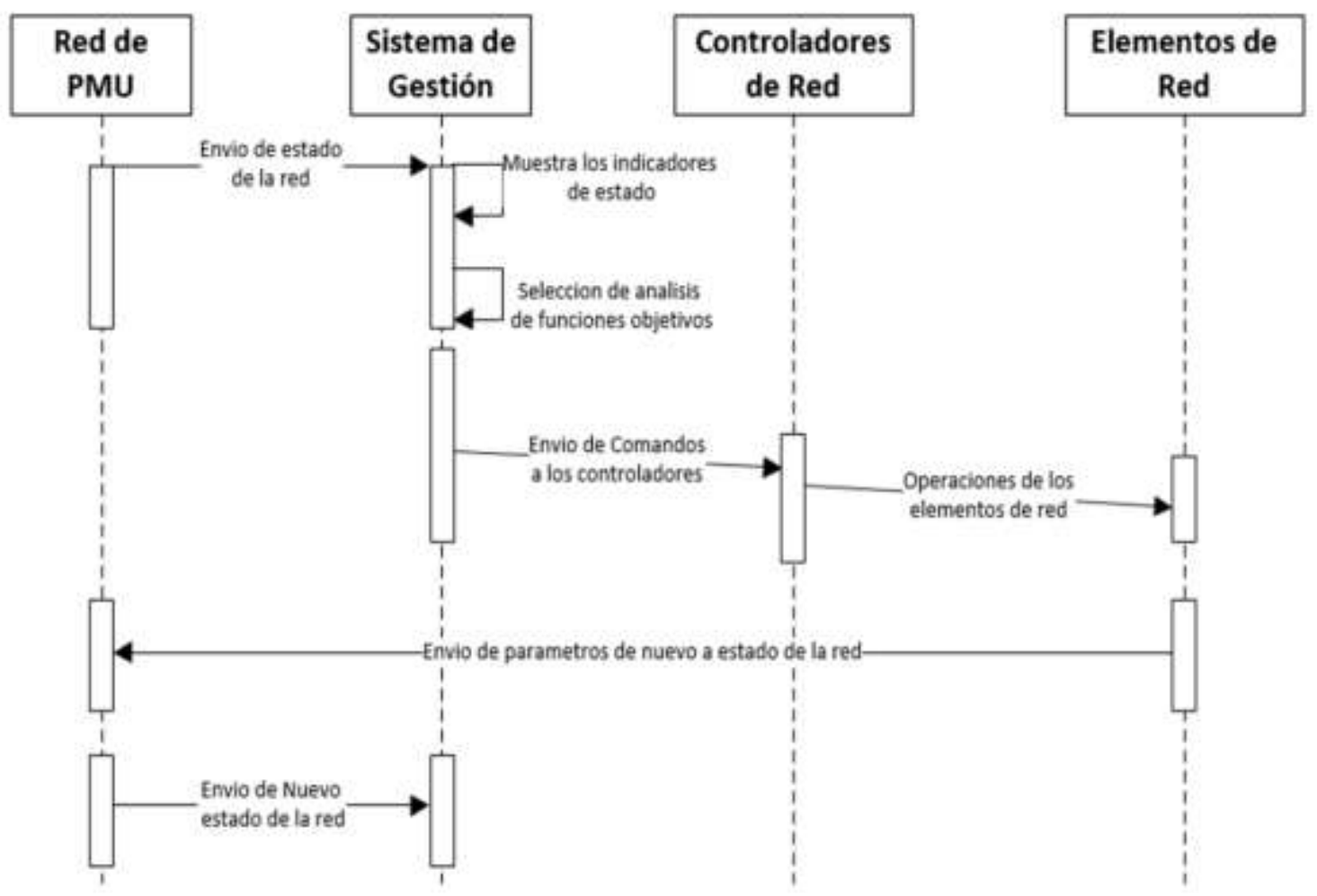

Fig. 5: Diagrama de secuencia para la operación del sistema de gestión propuesto.

\section{CONCLUSIONES}

El modelo de gestión propuesto se caracteriza por su integralidad en la forma de administrar la red, teniendo en cuenta el cumplimiento de funciones objetivo de diversa índole. Dichas funciones objetivo que abordan criterios técnicos, socio-económicos y medioambientales buscan que la gestión de la redes de distribución con inclusión de GD, no se limiten únicamente a un sentido estricto de la eficiencia energética del sistema, sino por el contrario busquen armonizar el desarrollo holístico de toda la red eléctrica y de los actores a quienes afecta directamente.

La utilización de una red de PMU, para el monitoreo de la red de distribución, y su posterior envío de información la base de datos pertinente, permite realizar análisis posteriores encaminados a determinar los mejores escenarios de inclusión de elementos de GD, y de cargas como los vehículos eléctricos, caracterizadas por su gran aleatoriedad en el arribo y permanencia en la red eléctrica. De igual forma la existencia de una red de PMU permite en determinados casos utilizar esquemas redundantes para aquellas eventualidades cuando una PMU salga de funcionamiento exista un respaldo de información sobre la sección o carga de red que se encontraba monitoreando.

El empleo de los diagramas de comportamiento UML, posibilita diseñar de forma genérica la estructura, e interacciones entre los eslabones funcionales de una red de distribución. Al ser diseños abiertos y genéricos, se permite fácilmente incluir nuevos parámetros en por ejemplo las funciones objetivo, o nuevo direccionamiento de los elementos de red, o nuevas estrategias de control para cumplir la tarea de gestión que se requiera en un determinado momento.

\section{REFERENCIAS}

Abdullah, M. "Calculation of Load Point Indices of Distribution Systems Due To Inserting a DG Device. In Radial Systems" (2011)

Abur, A., y Galvan, F., "Synchro-Phasor Assisted State Estimation (SPASE)". 2012 IEEE PES Innovative Smart Grid Technologies (ISGT), 02115, 1-2. http://doi.org/10.1109/ISGT.2012.6175559 (2012)

Alcalá, J., Charre, S., Durán, M., y Gudiño, J.,"Análisis del Convertidor CA/CD/CA (Back to Back) para la Gestión del Flujo de Potencia", Inf. Tec., 25(6), 109-116. http://doi.org/10.4067/S0718-07642014000600014 (2014) 
Andersen, P. B., Poulsen, B., Decker, M., Traeholt, C., y Ostergaard, J., "Evaluation of a Generic Virtual Power Plant framework using service oriented architecture". 2008 IEEE $2^{\text {nd }}$ International Power and Energy Conference, 1212-1217. http://doi.org/10.1109/PECON.2008.4762651 (2008)

Arias, L. A., Rivas, E., y Vega, C., "Propuesta de diseño para un centro integral de reciclaje con inclusión de fuentes alternativas de energía" (2014)

Arias L.A., García, A., y Rivas E., "Management model of distribution networks through UML and BPL" (2013)

Basile, F., Chiacchio, P., y Del Grosso, D.," A two-stage modelling architecture for distributed control of real-time industrial systems: Application of UML and Petri Net", Computer Standards \& Interfaces, 31(3), 528-538. http://doi.org/10.1016/j.csi.2008.03.021 (2009)

Basso, T., y DeBlasio, R., "IEEE Smart Grid Series of Standards IEEE 2030 (Interoperability) and IEEE 1547 (Interconnection) Status". Grid-Interop, 2030(September), 5-8, https://goo.gl/XSbXYT (2011)

Bayod-Rújula, A. "Future development of the electricity systems with distributed generation". Energy, 34(3), 377-383. http://doi.org/10.1016/j.energy.2008.12.008 (2009)

De Brabandere, K., Vanthournout, K., Driesen, J., Deconinck, G., y Belmans, R., "Control of Microgrids". 2007 IEEE Power Engineering Society General Meeting, (June), 1-7. http://doi.org/10.1109/PES.2007.386042 (2007)

Dietrich, K., Latorre, J. M., Olmos, L., y Ramos, A., "Modelling and assessing the impacts of self-supply and market-revenue driven Virtual Power Plants". Electric Power Systems Research, 119, $462-470$. http://doi.org/10.1016/j.epsr.2014.10.015 (2015)

Dolan, M. J., Davidson, E. M., Kockar, I., Ault, G. W., Mcarthur, S. D. J., y Member, S. "Active Power Flow Management" (2013)

Dulău, L. I., Abrudean, M., y Bică, D., "Distributed Generation Technologies and Optimization". Procedia Technology, 12, 687-692. http://doi.org/10.1016/j.protcy.2013.12.550 (2014)

Dulău, L. I., Abrudean, M., y Bică, D.,"SCADA Simulation of a Distributed Generation System with Storage Technologies". Procedia Technology, 19, 665-672. http://doi.org/10.1016/j.protcy.2015.02.094 (2015)

Dumitru, C.-D., y Gligor, A.,"A Management Application for the Small Distributed Generation Systems of Electric Power Based on Renewable Energy". Procedia Economics and Finance, 15(14), 1428-1437. http://doi.org/10.1016/S2212-5671(14)00608-X (2014)

Figueiredo, J., y Martins, J.,"Energy Production System Management - Renewable energy power supply integration with Building Automation System". Energy Conversion and Management, 51(6), 1120-1126. http://doi.org/10.1016/j.enconman.2009.12.020 (2010)

Gil González, W. J., Mora Flórez, J. J., y Pérez Londoño, S. M., "Análisis del procesamiento de los datos de entrada para un localizador de fallas en sistemas de distribución". (Spanish). Analysis of the Input Data Processing for Fault Location in Power Distribution Systems. (English), 18(41), 64-75. http://doi.org/http://dx.doi.org/10.14483/udistrital.jour.tecnura.2014.3.a05 (2014)

Giuntoli, M., y Poli, D., "Optimized thermal and electrical scheduling of a large scale virtual power plant in the presence of energy storages". IEEE Transactions on Smart Grid, 4(2), 942-955. http://doi.org/10.1109/TSG.2012.2227513 (2013)

Gómez, V. A., Peña, R. A., y Hernández, C.,"Identificación y localización de fallas en sistemas de distribución con medidores de calidad del servicio de energía eléctrica". Informacion Tecnologica, 23(2), 109-116. http://doi.org/10.4067/S0718-07642012000200013 (2012)

Inglis, S., Ault, G. W., Alarcon-Rodriguez, a., y Galloway, S. J.,"Multi-objective network planning tool for networks containing high penetrations of DER". Universities Power Engineering Conference (UPEC), 2010 45th International, 1-6. Retrieved from http://ieeexplore.ieee.org/xpl/freeabs_all.jsp?arnumber=5654393 (2010)

International Energy Agency. World Energy Outlook 2013. Agencia Internacional de Energía, 7. http://doi.org/10.1787/weo-2013-en (2013) 
Jagtap, K. M., y Khatod, D. K., "Loss allocation in radial distribution networks with various distributed generation and load models". International Journal of Electrical Power \& Energy Systems, 75, 173-186. http://doi.org/10.1016/j.ijepes.2015.07.042 (2016)

Kaabi, S. S. Al, Zeineldin, H. H., Member, S., y Khadkikar, V.,"Planning Active Distribution Networks Considering Multi-DG Configurations, 29(2), (2014)

Karabiber, A., Keles, C., Kaygusuz, A., y Alagoz, B. B., "An approach for the integration of renewable distributed generation in hybrid DC/AC microgrids". Renewable Energy, 52, $251-259$. http://doi.org/10.1016/j.renene.2012.10.041 (2013)

Lasseter, R. H.,"Microgrids and Distributed Generation". Journal of Energy Engineering, 133(3), $144-149$. http://doi.org/10.1061/(ASCE)0733-9402(2007)133:3(144) (2007)

Lo Prete, C., y Hobbs, B. F., "A cooperative game theoretic analysis of incentives for microgrids in regulated electricity markets". Applied Energy, 169, 524-541. http://doi.org/10.1016/j.apenergy.2016.01.099 (2016)

Masoum, M. a S., Ulinuha, a., Islam, S., y Tan, K.,"Hybrid passive filter design for distribution systems with adjustable speed drives". Proceedings of the International Conference on Power Electronics and Drive Systems, 1707-1712. http://doi.org/10.1109/PEDS.2007.4487938 (2007)

Mohamed, Y. A.,"New Control Algorithms for the Distributed Generation Interface in Grid-Connected and Microgrid Systems". Work (2008)

Ms, N. L., Ms., C. I., y Espiritu, J. F., "Evolutionary Agent Based Microstorage Management for a Hybrid Power System". Procedia Computer Science, 12(915), 350-355. http://doi.org/10.1016/j.procs.2012.09.083 (2012)

Mukhopadhyay, P., y Chawla, H. K., "Approach to make smart grid a reality". Proceedings of the 2014 International Conference on Advances in Energy Conversion Technologies - Intelligent Energy Management: Technologies and Challenges, ICAECT 2014, 77-82. http://doi.org/10.1109/ICAECT.2014.6757065 (2014)

Muñoz-Galeano, N., Cano-Quintero, J. B., y López-Lezama, J. M.,"Control de un Convertidor Elevador de Voltaje AC/DC Utilizando Balance de Potencias". Información Tecnológica, 27(2), 03-10. http://doi.org/10.4067/S0718-07642016000200002 (2016)

Nair, N.-K. C., y Zhang, L.,"SmartGrid: Future networks for New Zealand power systems incorporating distributed generation". Energy Policy, 37(9), 3418-3427. http://doi.org/10.1016/j.enpol.2009.03.025 (2009)

Nascimento, V. C. Do, Lambert-Torres, G., Costa, C. I. D. A., y Borges Da Silva, L. E., "Control model for distributed generation and network automation for microgrids operation". Electric Power Systems Research, 127, 151-159. http://doi.org/10.1016/j.epsr.2015.05.025 (2015)

Naus, J., Spaargaren, G., Van Vliet, B. J. M., y Van der Horst, H. M., "Smart grids, information flows and emerging domestic energy practices". Energy Policy, 68, 436-446.http://doi.org/10.1016/j.enpol.2014.01.038 (2014)

Pal, A., y Thorp, J. S.,"Co-ordinated control of inter-area oscillations using SMA and LMI". 2012 IEEE PES Innovative Smart Grid Technologies, ISGT 2012, 1-6. http://doi.org/10.1109/ISGT.2012.6175535 (2012)

Palizban, O., Kauhaniemi, K., y Guerrero, J. M.,"Microgrids in active network management - Part I: Hierarchical control, energy storage, virtual power plants, and market participation". Renewable and Sustainable Energy Reviews, 36, 428-439. http://doi.org/10.1016/j.rser.2014.01.016 (2014)

Patricia, G., Sepúlveda, L., David, C., y Londoño, S., "Estimación de los márgenes de estabilidad de tensión en un sistema de potencia usando redes neuronales" (2013)

Pudjianto, D., Pudjianto, D., Ramsay, C., Ramsay, C., Strbac, G., y Strbac, G., "Virtual power plant and system integration of distributed energy resources". Renewable Power Generation, IET, 1(1), 10-16. http://doi.org/10.1049/iet-rpg (2007)

Ramírez A., Chica A., y Arias L.A.,"MicroRed inteligente sustentable de biogás para zona no interconectada" (2013) 
Ren, J., Kezunovic, M., y Guan, Y., "Verifying interoperability and application performance of PMUs and PMUenabled IEDs". IEEE Power and Energy Society General Meeting, 1-3. http://doi.org/10.1109/PESGM.2012.6345385 (2012)

Ruiz-Romero, S., Colmenar-Santos, A., Mur-Pérez, F., y López-Rey, Á., "Integration of distributed generation in the power distribution network: The need for smart grid control systems, communication and equipment for a smart city - Use cases". Renewable and Sustainable Energy Reviews, 38, $223-234$. http://doi.org/10.1016/j.rser.2014.05.082 (2014)

Ruschmann, M. C., Wu, N. E., y Huang, J., "Redundancy architecture design of PMU networks for availability of synchrophasors". 2012 IEEE PES Innovative Smart Grid Technologies, ISGT 2012. http://doi.org/10.1109/ISGT.2012.6175654 (2012)

Sadeghi, A., Larimian, T., y Molabashi, A., "Evaluation of Renewable Energy Sources for Generating Electricity in Province of Yazd: A Fuzzy Mcdm Approach". Procedia - Social and Behavioral Sciences, 62, $1095-1099$. http://doi.org/10.1016/j.sbspro.2012.09.187 (2012)

Schäfer, M., Gretzschel, O., Schmitt, T. G., y Knerr, H.,"Wastewater Treatment Plants as System Service Provider for Renewable Energy Storage and Control Energy in Virtual Power Plants - A Potential Analysis". Energy Procedia, 73, 87-93. http://doi.org/10.1016/j.egypro.2015.07.566 (2015)

Soshinskaya, M., Crijns-Graus, W. H. J., Guerrero, J. M., y Vasquez, J. C.,"Microgrids: Experiences, barriers and success factors". Renewable and Sustainable Energy Reviews, 40, 659-672. http://doi.org/10.1016/j.rser.2014.07.198 (2014)

Sowa, T., Krengel, S., Koopmann, S., y Nowak, J., "Multi-criteria operation strategies of power-to-heat-Systems in virtual power plants with a high penetration of renewable energies". Energy Procedia, 46, $237-245$. http://doi.org/10.1016/j.egypro.2014.01.178 (2014)

Tazvinga, H., Xia, X., y Zhu, B.,"Optimal energy management strategy for distributed energy resources". Energy Procedia, 61, 1331-1334. http://doi.org/10.1016/j.egypro.2014.11.1093 (2014)

Tse, N. C. F., y Zhou, L.,"Detection of voltage variations due to distributed energy resources". 2009 IEEE Power and Energy Society General Meeting, PES '09, (2), 2-6. http://doi.org/10.1109/PES.2009.5275580 (2009)

Vaschetti, J. C., Magnago, F., y Sauchelli, V. H.,"Control automático de voltaje en sistemas eléctricos de potencia basado en sistemas expertos". Informacion Tecnologica, 23(5), 69-84. http://doi.org/10.4067/S0718$07642012000500008(2012)$

Vega, C., Florez, O., Arias, L. A. y Rivas, E.,"Distribution networks management system with multi-target operations using UML". 2014 leee Andescon, 1-1. http://doi.org/10.1109/ANDESCON.2014.7098543 (2014)

Ye, D., Zhang, M., y Sutanto, D.,"Decentralised dispatch of distributed energy resources in smart grids via multiagent coalition formation". Journal of Parallel and Distributed Computing, 83, 30-43. http://doi.org/10.1016/j.jpdc.2015.04.004 (2015)

You, S., y Segerberg, H.,"Integration of $100 \%$ micro-distributed energy resources in the low voltage distribution network: A Danish case study". Applied Thermal Engineering, 71(2), 797-808. http://doi.org/10.1016/j.applthermaleng.2013.11.039 (2013)

Zhang, X., Member, S., Sharma, R., y He, Y.,"Optimal Energy Management of a Rural Microgrid System Using Multi-objective" (2011) 
